\title{
La Cultura de Movilidad y el Uso de la Bicicleta
}

\section{The Mobility Culture and the Use of the Bicycle}

Hermes Rigoberto Auris Céspedes ${ }^{1}$

\section{RESUMEN}

Objetivo: Determinar la relación entre la cultura de la movilidad y la sensibilidad en el uso de la bicicleta en estudiantes de II.EE. emblemáticas de la UGEL 03 de Lima inmersos en el proyecto educativo 'Al cole en bici' en el año 2017. Material y Métodos: El diseño utilizado en la investigación; fue descriptivo correlacional; este estudio científico se clasifica como una investigación transversal. La población estuvo conformada por estudiantes de las instituciones educativas de la UGEL 03, de donde se seleccionaron 120 estudiantes como muestra a los cuales se les aplicó los instrumentos de investigación; estos instrumentos fueron dos encuestas con gradiente de 5, de 24 y 12 ítems respectivamente; la confiabilidad, de dichos instrumentos, se aseguró por medio del coeficiente Alfa de Cronbach (0.902, 0.726 respectivamente); la validez de los instrumentos se hizo por medio del juicio de expertos. Para el análisis estadístico, se utilizó el software estadístico SPSS 24. Resultados: Existe una relación baja entre el nivel de la cultura de movilidad y el nivel de sensibilidad en el uso de la bicicleta, mostrando que la variación en el uso de la bicicleta implica un cambio menor en la cultura ciclista, mostrando un impacto del proyecto relativamente medio del proyecto "Al cole en bici". Se establece que existe una relación directa entre la cultura de la movilidad y la sensibilidad en el uso de la bicicleta en estudiantes de II.EE. emblemáticas de la UGEL 03 de Lima inmersos en el proyecto educativo 'Al cole en bici' en el año 2017. Conclusión: Se ha determinado que el nivel de sensibilidad en el uso de la bicicleta tiene un desarrollo medio, por tanto es posible promover el uso de la bicicleta por medio de la formación en la cultura de la movilidad de tal manera que la sensibilidad para el uso de la bicicleta sea mucho más abierta.

Palabras clave: Proyectos, ciclismo, educación básica y evaluación.

\section{ABSTRACT}

Objective: To determine the relationship between the culture of mobility and sensitivity in the use of the bicycle in II.EE students. emblematic of the UGEL 03 of Lima immersed in the educational project 'Al cole en bici' in 2017. Material and Methods: The design used in the research; it was descriptive correlational; This scientific study is classified as a cross-sectional investigation. The population was made up of students from the educational institutions of the UGEL 03, from which 120 students were selected as a sample to which the research instruments were applied; These instruments were two surveys with a gradient of 5,24 and 12 items respectively; the reliability of these instruments was ensured by means of Cronbach's alpha coefficient $(0.902,0.726$ respectively); the validity of the instruments was made through expert judgment. For the statistical analysis, the SPSS 24 statistical software was used. Results: There is a low relationship between the level of mobility culture and the level of sensitivity in the use of the bicycle, showing that the variation in the use of the bicycle implies a minor change in the cycling culture, showing an impact of the project relatively average of the project "Al cole en bici". It is established that there is a direct relationship between the culture of mobility and sensitivity in the use of bicycles in II.EE students. emblematic of the UGEL 03 of Lima immersed in the educational project 'Al cole en bici' in 2017. Conclusion: It has been determined that the level of sensitivity in the use of the bicycle has a medium development, therefore it is possible to promote the use of the bicycle through training in the culture of mobility in such a way that the sensitivity for the use of the bicycle is much more open.

KEYWORDS: Projects, cycling, basic education and evaluation

\section{INTRODUCCIÓN}

Existen programas que abarcan el aspecto de la movilidad, pero sus repercusiones no han hecho eco; solo se ha reducido al desplazamiento de medios para transportarse y no han logrado un adecuado modelo de movilidad. Es por ello que se debe reconstruir la ciudad de Lima a través de una relación lógica en un espacio que le pertenezca al peatón, a la bicicleta y los medios de transportes masivos; se debe realizar una planificación urbana apropiada. Es así que se plantea una idea que emerge desde el ámbito escolar pues el desarrollo de los estudiantes de educación básica regular no solo debe estar orientado al afianzamiento y desarrollo de la motricidad, sino también se debe poner énfasis en la cultura de la vida saludable, con mayor énfasis en las nuevas generaciones, las cuales está expuestas a aprendizajes que inducen al sedentarismo producto del desarrollo tecnológico. El incremento de las nuevas herramientas tecnológicas brindan juegos y softwares que no orientan hacia las actividades saludables dejando de lado las actividades físicas y deportivas, situación que, aunada a la proliferación del consumo de alimentos poco saludables conllevan al incremento de la obesidad en los estudiantes de formación escolar (La República, 2015) y en la población en general (El Comercio, 2017). Esta situación se va agravando según se incrementa la tecnología y la desnaturalización del trabajo, aumentando y ahondando las circunstancias desfavorables para políticas de salud. Por otro lado, en muchas ciudades del Perú se observa un gran incremento de la congestión vehicular y la proliferación de vehículos motorizados en menoscabo del medio ambiente y la salud de las personas, es así que "el desarrollo de medios de transporte urbanos que han acompañado el crecimiento de las ciudades ha estado signado por una fuerte presencia de transportes motorizados, y en gran parte de índole privada. Incluso

Recibido:03/02/2020 - Aprobado:15/02/2020

Universidad Nacional Mayor de San Marcos (Lima - Perú). 
los proyectos públicos de movilidad urbana han estado con frecuencia asociados a transporte motorizado como autobuses o trenes" (Noel, Santos, \& Taks, 2013, p. 2).

Como antecedentes, primero se abordó la cultura de la movilidad; ante ello Camacho (2017) menciona, desde su postura antropológica, que "es la manera en cómo los seres humanos viven, sienten, piensan y actúan en, desde y para lo cotidiano de los espacios de movilización y desplazamiento" ( $p$ 16). Thema Equipo Editorial S.A. (2000) primero define el concepto de cultura, el cual es un punto de partida importante para poder entender mejor la cultura de movilidad; la menciona como: "un conjunto de costumbres, conocimientos, y grado de desarrollo artístico, científico, etc., en una época o grupo social" (p. 289). Del mismo modo, el Fondo de Prevención Vial (2006) la define como: "un conjunto de costumbres, acciones y reglas mínimas compartidas que generan sentido de pertenencia, facilitan la convivencia ciudadana y conducen al respeto del patrimonio común y al reconocimiento de los derechos y los deberes ciudadanos" (p. 3). Pardo (2006) sostiene que "la cultura de movilidad permite generar conciencia ciudadana sobre transporte urbano sostenible" (p. 27). Ballester y Peiró (2008) mencionan que: "La organización de la sociedad requiere en gran medida la movilidad como un elemento esencial, y su mejora ha tenido muchos efectos beneficiosos para la población. Por último, Alcántara (2010) señala que tiene que practicarse más la cultura de la movilidad porque: "Ios medios de transporte que usan motor, se relacionan con el 'efecto barrera', de ahí que, cuando las relaciones sociales se ven afectadas. El resultado es un gran impacto en la vida de las personas de las distintas localidades, este hecho (casi seguro) puede hacerse más severo en los próximos años, ya que, la motorización, va en aumento, principalmente, en los países que se están pasando por el proceso de desarrollo. En lo que respecta al uso de bicicleta Rodríguez (2017) menciona que: "es una necesidad creciente en el país y en todos los demás, de ahí en América Latina las autoridades urbanas están prestando cada vez más interés en promover la bicicleta como medio de transporte cotidiano y en rescatar el valor que ha tenido por décadas como engranaje de la vida en comunidad" (p. 6). Caballero(2014), por su parte, señala que: "el uso masivo de transportes sustentables como la bicicleta, no sólo requieren cambios en la infraestructura de las ciudades sino un cambio en el comportamiento. Morales (2011) menciona que "las bicicletas son el único medio de transporte que no consume energía externa al organismo y que es el vehículo terrestre más económico del mundo, pues es el que menos energía consume en relación con la distancia recorrida. Ahora, en lo que se refiere al nivel de sensibilidad en el uso de la bicicleta, Monzón, Rondinella y La Paix(2009) señalan que deben existir acciones orientadas para potenciar el uso de los diferentes modos sobre el uso de la bicicleta. Es por ello que resulta importante identificar cuáles son estos diferentes usos que se puede dar a la bicicleta, los cuales, según los autores son la de usar la bicicleta como un elemento de traslado, como como elemento para la recreación y como elemento de deporte." (p. 37). Dora, Hosking, Mudu, Fletcher y Elaine (2011) mencionan que "el uso de la bicicleta es importante ante un determinante contexto social. Este fenómeno social es importante ya que según sea la preferencia social, es decir, si la sociedad considera que, por ejemplo, para ella es más importante el uso de la bicicleta para el deporte, sobre este uso es que pondrá todas sus acciones, las cuales estarán orientadas a incentivar el desarrollo de la bicicleta y el deporte". Por último, Castellar, Pradas, Rapún, Coll y Pérez(2013) mencionan que: "la utilización de la bicicleta no solo se circunscribe al entorno urbano, sino que puede extenderse al medio natural próximo, ofreciéndonos un nuevo espacio docente lleno de recursos y posibilidades.

Por último, se tuvo como objetivo fundamental analizar y explicar la influencia de la cultura de movilidad en el uso de la bicicleta en estudiantes de II.EE. emblemáticas de la UGEL 03 de Lima inmersos en el proyecto educativo 'Al cole en bici' en el año 2017.

\section{MATERIALES Y MÉTODOS}

La metodología usada en el presente trabajo fue cuantitativa; ambas variables pasaron por proceso de operacionalización en el cual se les adjudicó valores numéricos.

Participaron en la encuesta 120 estudiantes que conformaron la muestra, organizados por dimensiones de acuerdo a la operacionalización de las variables. Para el análisis estadístico, se usó el software estadístico SPSS 24.

El diseño de investigación es descriptivo correlacional ya que se buscó determinar el nivel de correlación de las variables por medio del coeficiente de correlación de Pearson, paralelamente se muestra la descripción de los resultados de ambas variables.

La investigación se clasifica como una investigación transversal:

$$
M=O x-O y
$$

Donde:

$$
M=\text { Muestra }
$$

$\mathrm{O}=$ Relación

$X=$ Nivel de sensibilidad en el uso de la bicicleta

$\mathrm{Y}=$ Nivel de la cultura de movilidad

Las variables se pueden clasificar de la siguiente manera:

Variable independiente: El nivel de sensibilidad en el uso de la bicicleta $(X)$

Variable dependiente: El nivel de la cultura de movilidad (Y) 
Variables extrañas:

Podemos señalar las siguientes:

Disponibilidad de tiempo

\section{Tipo de bicicleta \\ Situación socioeconómica \\ Localización de vivienda}

\section{Tabla 1}

Operacionalización de la variable:

Nivel de sensibilidad en el uso de la bicicleta $(X)$.

\begin{tabular}{|c|c|c|c|c|}
\hline VARIABLE & $\begin{array}{l}\text { DIMENSIONES/ } \\
\text { FACTORES }\end{array}$ & INDICADORES & ÍTEM & PUNTAJE \\
\hline & $\mathrm{x}_{1}:$ Recreacion & $\begin{array}{l}\text { Tiempo de uso. } \\
\text { constancia de uso. } \\
\text { preferencia de uso. } \\
\text { costo de oportunidad. }\end{array}$ & $\begin{array}{l}1 \\
2 \\
3 \\
4\end{array}$ & \\
\hline \multirow[t]{2}{*}{$\begin{array}{l}\text { El nivel de } \\
\text { sensibilidad } \\
\text { en el uso de } \\
\text { la bicicleta }\end{array}$} & $\mathrm{x}_{2}$ :Deporte & $\begin{array}{l}\text { Tiempo de uso. } \\
\text { constancia de uso. } \\
\text { preferencia de uso. } \\
\text { costo de oportunidad. }\end{array}$ & $\begin{array}{l}5 \\
6 \\
7 \\
8\end{array}$ & {$[1-5]$} \\
\hline & $\mathrm{x}_{3}:$ Transporte & $\begin{array}{l}\text { Tiempo de uso. } \\
\text { constancia de uso. } \\
\text { preferencia de uso. } \\
\text { costo de oportunidad. }\end{array}$ & $\begin{array}{l}9 \\
10 \\
11 \\
12\end{array}$ & \\
\hline
\end{tabular}

Tabla 2

Operacionalización de la variable:

Nivel de la cultura de movilidad (Y).

\begin{tabular}{|c|c|c|c|c|c|}
\hline VARIABLE & $\begin{array}{l}\text { DIMENSIONES/ } \\
\text { FACTORES }\end{array}$ & INDICADORES & ORIENTACIÓN & ÍTEM & PUNTAJE \\
\hline & $\begin{array}{c}Y_{1}: \text { Educacion } \\
\text { vial }\end{array}$ & $\begin{array}{l}\text { Espacio para circular. } \\
\text { Circulación en carriles } \\
\text { compartidos. } \\
\text { Traslado de personas o } \\
\text { paquetes. } \\
\text { Trasladar por vías exclusivas } \\
\text { de transporte motorizado. } \\
\text { Respeto a la señales de } \\
\text { tránsito. } \\
\text { Uso de la ciclo vías. } \\
\text { Compartir de manera } \\
\text { responsable la circulación } \\
\text { por vías comunes. } \\
\text { Relación con otros vehículos } \\
\text { en movimiento. }\end{array}$ & $\begin{array}{l}\mathrm{P} \\
\mathrm{P} \\
\mathrm{N} \\
\mathrm{N} \\
\mathrm{P} \\
\mathrm{P} \\
\mathrm{P} \\
\mathrm{N} \\
\mathrm{P} \\
\mathrm{N}\end{array}$ & $\begin{array}{l}1 \\
2 \\
3 \\
4 \\
\\
5 \\
6 \\
6 \\
7 \\
8 \\
9 \\
\\
10\end{array}$ & \\
\hline $\begin{array}{l}\text { El nivel de la } \\
\text { cultura de } \\
\text { movilidad (Y) }\end{array}$ & $\begin{array}{l}Y_{2}: \text { Medida de } \\
\text { seguridad }\end{array}$ & $\begin{array}{l}\text { Utilización de implementos } \\
\text { de seguridad. } \\
\text { Lugar de desplazamiento. } \\
\text { Contar con reflectantes. } \\
\text { contar con luces. } \\
\text { contar con timbre o bocina. }\end{array}$ & $\begin{array}{l}P \\
N \\
P \\
P \\
P \\
P\end{array}$ & $\begin{array}{l}11 \\
12 \\
13 \\
14 \\
15 \\
16\end{array}$ & [1-5] \\
\hline & $\begin{array}{l}Y_{3}: \text { Buenas } \\
\text { practicas }\end{array}$ & $\begin{array}{l}\text { La bicicleta puede hacer } \\
\text { ruidos. } \\
\text { Contar con kit de emergencia } \\
\text { (camara, llave, allen, inflador, } \\
\text { etc.) } \\
\text { Contar con herramientas } \\
\text { para hacer el mantenimiento } \\
\text { preventivo. } \\
\text { Conocimiento para hacer } \\
\text { mantenimiento preventivo. } \\
\text { Ropa e implementos } \\
\text { adecuados. } \\
\text { Verificación rápida de buen } \\
\text { estado: frenos, dirección, } \\
\text { presión de llantas. } \\
\text { mantenimiento constantes. }\end{array}$ & $\begin{array}{l}\mathrm{N} \\
\mathrm{P} \\
\mathrm{P} \\
\mathrm{P} \\
\mathrm{P} \\
\mathrm{P} \\
\mathrm{N}\end{array}$ & $\begin{array}{l}17 \\
18 \\
19 \\
20 \\
21 \\
22 \\
23 \\
24\end{array}$ & \\
\hline
\end{tabular}

\section{RESULTADOS}

A continuación, se presenta las variables que formaron parte de la investigación así se tiene; "El nivel de sensibilidad en el uso de la bicicleta (X)" y "El nivel de la cultura de movilidad (Y)"; Los resultados que se obtuvieron al hacer el análisis estadístico son: 
Tabla 3

Frecuencias del nivel de sensibilidad en el uso de la bicicleta $(X)$

\begin{tabular}{|c|c|c|c|c|c|}
\hline \multicolumn{2}{|c|}{ FRECUENCIAS } & FRECUENCIA & PORCENTAJE & $\begin{array}{l}\text { PORCENTAJE } \\
\text { VÁLIDO }\end{array}$ & $\begin{array}{l}\text { PORCENTAJE } \\
\text { ACUMULADO }\end{array}$ \\
\hline V & {$[12 ; 24>$} & 25 & 20,8 & 20,8 & 20,8 \\
\hline$\stackrel{A}{L}$ & {$[24 ; 36>$} & 57 & 47,5 & 47,5 & 68,3 \\
\hline I & {$[36 ; 48>$} & 32 & 26,7 & 26,7 & 95,0 \\
\hline D & {$[48 ; 60]$} & 6 & 5,0 & 5,0 & 100,0 \\
\hline & TOTAL & 120 & 100,0 & 100,0 & \\
\hline
\end{tabular}

Tabla 4

Estadísticos de nivel de sensibilidad en el uso de la bicicleta $(X)$

\begin{tabular}{lrr}
\hline $\mathbf{N}$ & $\begin{array}{c}\text { VALIDO } \\
\text { PERDIDO }\end{array}$ & $\mathbf{1 2 0}$ \\
& & \\
\hline Media & & 31,03 \\
Mediana & 31,00 \\
Moda & & 29 \\
Desviación estándar & 9,505 \\
Coeficiente de variación & & 0,306 \\
Varianza & & 90,344 \\
Asimetría & & 0,025 \\
& & 0,021 \\
Error estándar de asimetría & & \\
Curtosis & & $-0,405$ \\
Error estándar de curtosis & & 0,438 \\
Minimo & & 12 \\
Maximo & & 52 \\
& & \\
Percentiles & & 25,00 \\
& 25 & 31,00 \\
& 50 & \\
\hline
\end{tabular}

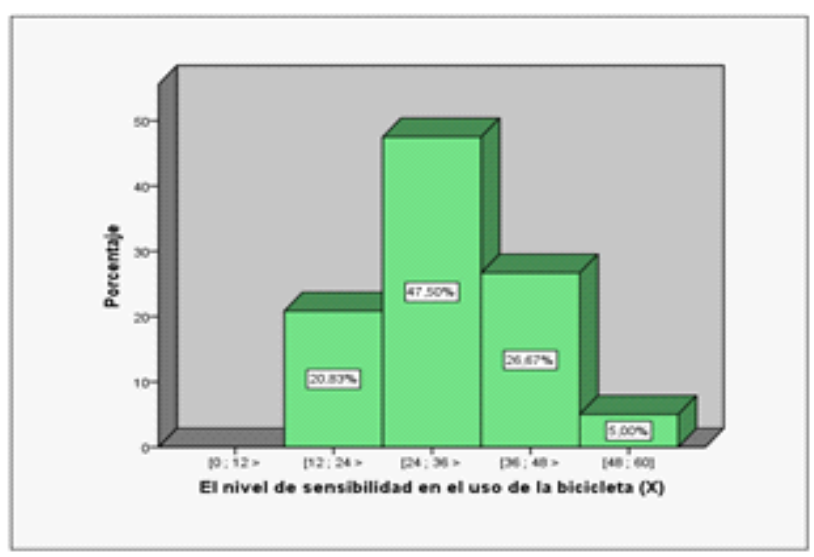

Figura 1. Histograma en porcentaje del nivel sensibilidad en el uso de la bicicleta(X).

Se observó que una gran $47,5 \%$ de los estudiantes evaluados se encontraron en el intervalo [24;36> clasificado como medio, además la media fue de 31,03 puntos con una desviación estándar de 9,505, esto lleva a un coeficiente de variación (CV) 30,6\% indicando una relativa homogeneidad de los datos. El coeficiente de asimetría fue de 0,025 indicando que la distribución es asimétrica positiva y según la curtosis que toma un valor de $-0,405$ indicó que la distribución es platocúrtica.

Se puede observar que la variable "Nivel de sensibilidad en el uso de la bicicleta (X)" tuvo un desarrollo medio, mostrando que los estudiantes, respecto de esta variable, tiene un desarrollo donde una cantidad importante de ellos toman valores entre el intervalo [24;36>, lo cual indica que el proyecto "Al cole en bici” tuvo un impacto medio.

Tabla 5

Frecuencias del nivel de la cultura de movilidad (Y)

\begin{tabular}{llllll}
\hline FRECUENCIAS & FRECUENCIA & $\begin{array}{c}\text { PORCENTAJE PORCENTAJE } \\
\text { VÁLIDO }\end{array}$ & $\begin{array}{l}\text { PORCENTAJE } \\
\text { ACUMULADO }\end{array}$ \\
\hline V & {$[48 ; 72>$} & 64 & 53,3 & 53,3 & 53,3 \\
A & {$[72 ; 96>$} & 52 & 43,3 & 43,3 & 96,7 \\
L & {$[96 ; 120]$} & 4 & 3,3 & 3,3 & 100,0 \\
I & & & & & \\
D & & & 100,0 & 100,0 & \\
O & TOTAL & 120 & 100 & \\
& & & & & \\
\hline
\end{tabular}

Tabla 6

Estadísticos del nivel de la cultura de movilidad (Y)

\begin{tabular}{lrr}
\hline $\mathbf{N}$ & $\begin{array}{r}\text { VALIDO } \\
\text { PERDIDO }\end{array}$ & $\mathbf{1 2 0}$ \\
& & $\mathbf{0}$ \\
\hline Media & & 71,61 \\
Mediana & 71,00 \\
Moda & 70 \\
Desviación estándar & 11,659 \\
Coeficiente de variación & 0,163 \\
Varianza & 135,921 \\
Asimetría & 0,276 \\
Error estándar de asimetría & & 0,221 \\
& & \\
Curtosis & & $-0,299$ \\
Error estándar de curtosis & & 0,438 \\
Minimo & 48 \\
Maximo & & 104 \\
& & \\
Percentiles & & 63,00 \\
& 25 & 71,00 \\
& 50 & 78,75
\end{tabular}

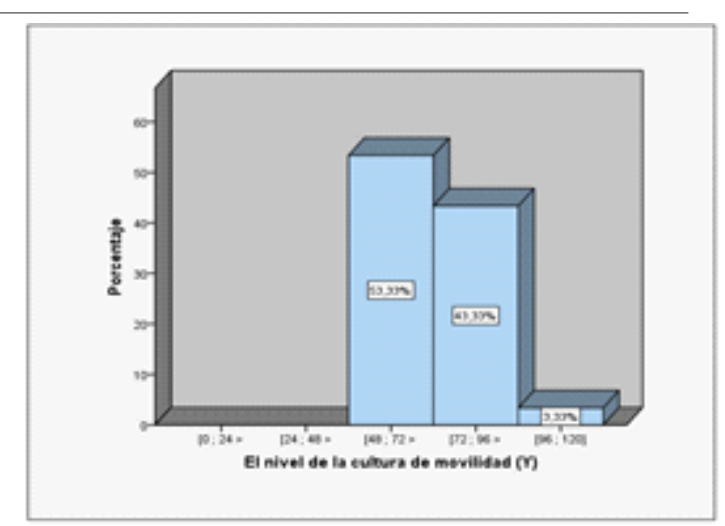

Figura 2. Histograma en porcentaje del nivel de la cultura de movilidad $(Y)$.

Se observa que un $53,33 \%$ de los estudiantes evaluados se encuentran en el intervalo [48; 72> clasificado como medio, además la media fue de 71,61 puntos con una desviación estándar de 11,659, esto lleva a un coeficiente de variación (CV) $16,3 \%$ indicando una alta homogeneidad de los datos. El coeficiente de asimetría fue de 0,276 indicando que la distribución es asimétrica positiva y según la curtosis que toma un valor de $-0,299$ indica que la distribución es platocúrtica.

Podemos observar que la variable "El nivel de la cultura de movilidad (Y)" tiene un desarrollo medio, mostrando que los estudiantes, respecto de esta variable, tiene un desarrollo donde una gran cantidad de ellos toman valores entre el intervalo [48; 72 > de un máximo de 120 puntos, lo cual indica 
que el proyecto "Al cole en bici" tiene un impacto medio en esta variable.

Con respecto a las hipótesis, se desprenden la hipótesis nula $\left(\mathrm{H}_{0}\right)$ y la hipótesis alterna $\left(\mathrm{H}_{\mathrm{a}}\right)$ :

\section{Hipótesis Nula $\left(H_{0}\right)$ :}

El nivel de la cultura de movilidad y el nivel de sensibilidad en el uso de la bicicleta en estudiantes de II.EE. emblemáticas de la UGEL 03 de Lima inmersos en el proyecto educativo "Al cole en bici" en el año 2017 son variables dependientes.

\section{Hipótesis Alterna $\left(H_{a}\right)$ :}

El nivel de la cultura de movilidad y el nivel de sensibilidad en el uso de la bicicleta en estudiantes de II.EE. emblemáticas de la UGEL 03 de Lima inmersos en el proyecto educativo "AI cole en bici" en el año 2017 son variables independientes. Se procedió a realizar el análisis correlacional correspondiente considerando la regla teórica para la toma de decisión es Si el Valor $p \geq 0.05$ se acepta la Hipótesis Alterna (Ha). Si el Valor $p<0.05$ se acepta la Hipótesis nula (Ho), si el $p<0.01$ entonces se dice que la relación entre ambas variables es altamente significativa.

La contrastación de hipótesis utiliza la correlación coeficiente de Pearson entre las variables el nivel de la cultura de movilidad y el nivel de sensibilidad en el uso de la bicicleta:

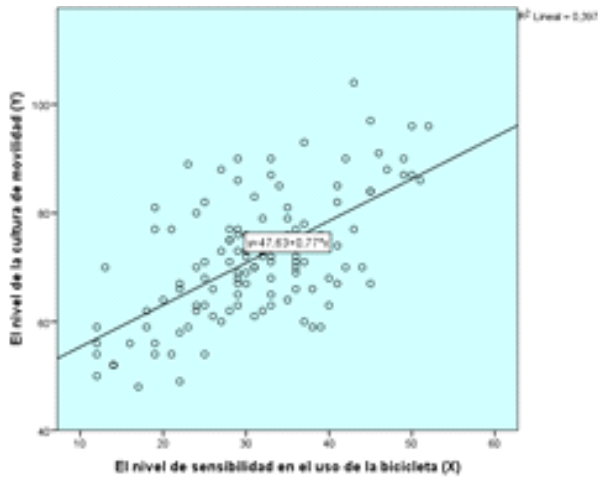

Figura 3 Diagrama de dispersión entre el nivel sensibilidad en el uso de la bicicleta $(\mathrm{X})$ y del nivel de la cultura de movilidad (Y)

Tabla 7

Correlación entre las variables $X$ e $Y$

\begin{tabular}{|c|c|c|c|}
\hline & & $\begin{array}{l}\text { EL NIVEL DE } \\
\text { SENSIBILIDADEN EL } \\
\text { USO DE LA } \\
\text { BICICLETA (X) }\end{array}$ & $\begin{array}{l}\text { EL NIVEL DE LA } \\
\text { CULTURA DE } \\
\text { MOVILIDAD(Y) }\end{array}$ \\
\hline \multirow{3}{*}{$\begin{array}{l}\text { EL NIVEL DE } \\
\text { SENSIBILIDADE } \\
\text { N EL USO DE } \\
\text { LA BICICLETA } \\
\text { (X) }\end{array}$} & $\begin{array}{l}\text { Correlación de } \\
\text { pearson }\end{array}$ & 1 &, $395^{* \star}$ \\
\hline & Sig.(bilateral) & & 0,000 \\
\hline & $N$ & 120 & 12 \\
\hline \multirow{3}{*}{$\begin{array}{l}\text { EL NIVEL DE } \\
\text { LA CULTURA } \\
\text { DE } \\
\text { MOVILIDAD(Y) }\end{array}$} & $\begin{array}{l}\text { Correlación } \\
\text { depearson }\end{array}$ &, $395^{* *}$ & 1 \\
\hline & Sig.(bilateral) & 0,000 & \\
\hline & $\mathbf{N}$ & 120 & 120 \\
\hline
\end{tabular}

Se obtuvieron los siguientes resultados:

$\begin{array}{lll}\text { VALOR- } & < & \text { VALOR X } \\ 0.0 & < & 0.05 \\ \mathrm{H}_{0}=\text { Se acepta } & & \end{array}$

Por lo tanto, se afirma que el nivel de la cultura de movilidad y el nivel de sensibilidad en el uso de la bicicleta en estudiantes de II.EE. emblemáticas de la UGEL 03 de Lima inmersos en el proyecto educativo "Al cole en bici" en el año 2017 son variables dependientes. Ya que el coeficiente de significancia (0.0) es menor que el parámetro de confianza 0.05 y el coeficiente de correlación de Pearson es muy bajo igual a 0,395.

\section{DISCUSIÓN}

Existe muchas investigaciones orientadas al uso de la bicicleta como es el caso de Rivera(2015) donde se plantea que la solución para las diversas problemáticas del transporte es el uso de la bicicleta, como una propuesta de desarrollo viable para la ciudad de Lima; estos mismos estudios se hicieron a nivel internacional en otros países como son el caso de Suero (2010) en Colombia, así también en México Hilario, Núñez, y Paniagua(2015) muestran como alternativa el uso de la bicicleta como parte de la solución a la problemática del transporte, así también existen investigaciones que muestran la eficiencia del uso del transporte como el caso en la ciudad de Madrid de la investigación hecha por Fernández (2012). Sin embargo, no ha sido posible encontrar investigaciones que estudien las variables el nivel de sensibilidad en el uso de la bicicleta, tampoco, el nivel de la cultura de movilidad. Cuando una investigación es del tipo exploratorio no es factible encontrar antecedentes que cubran investigaciones anteriores; esto es factible puesto que en el presente estudio busca identificar la sensibilidad en el uso de la bicicleta que, como se ha observado, es la forma de ver la disposición de los estudiantes de educación básica regular para usar este medio de transporte, la cual, no es una realidad muy estudiada, la cultura de la movilidad no ha sido atendida por los investigadores, donde lo que se quiere determinar el conocimiento de cómo debe desplazarse el ciclista en el medio urbano considerando aspectos de seguridad que evitarían complicaciones en el proceso de la movilidad; por tanto, es necesario que se pueda desarrollar investigaciones que aporten a la investigación de estas variables.

Para un contraste suficientemente aceptable, los resultados obtenidos deberían ser comparados y contrastados con los resultados del proyecto "Al cole en bici" (Municipalidad Metropolitana de Lima, 2017), sin embargo, esto no es posible ya que no se ha logrado acceder a los resultados del proyecto

\section{CONCLUSIONES}

Existe una relación directa entre la cultura de la movilidad y la sensibilidad en el uso de la bicicleta en estudiantes de II.EE. emblemáticas de la UGEL 03 de Lima inmersos en el proyecto educativo 'Al cole en bici' en el año 2017.

El nivel de sensibilidad en el uso de la bicicleta tuvo un desarrollo medio y el nivel de la cultura de movilidad un desarrollo alto.

Muy pocos estudiantes utilizan la bicicleta como medio de transporte diario.

Es posible promover el uso de la bicicleta por medio de la 
formación en la cultura de la movilidad de tal manera que la sensibilidad para el uso de la bicicleta sea mucho más abierta.

\section{AGRADECIMIENTOS}

El presente trabajo no hubiese culminado sin los valiosos aportes de la Dra. Jesahel Vildoso, mi asesora y maestra de la universidad, mi agradecimiento y consideración hacia ella serán eternos. Una fuente de motivación importante fue la Conferencia Hábitat III, la cual, apoyándose en el ODS 11, busca "lograr que las ciudades y los asentamientos humanos sean inclusivos, seguros, resilientes y sostenibles".

\section{REFERENCIAS BIBLIOGRÁFICAS}

Alcántara, E. (2010). Análisis de la movilidad urbana. Espacio, medio ambiente y equidad. Bogotá: Corporación Andina de Fomento.

Ballester, F., y Peiró, R. (2008). Transporte, medio ambiente y salud. SESPAS, 53-64.

Caballero, R., Alba, P. F., y Jakovcevic, A. (2014). Uso de la bicicleta como medio de transporte: influencia de los factores psicológicos. Una revisión de la literatura. Psico, 45(3), 316-327.

Camacho, G. (28 de agosto de 2017). ¿Qué es cultura vial? Obtenido de culturavial.net: https://culturavial.net/quees-cultura-vial/

Castellar, C., Pradas, F., Rapúm, M., Coll, I., y Pérez, S. (2013). Aula en bici: un proyecto longitudinal de intervención docente en Ed. Primaria. Retos: nuevas tendencias en educación física, deporte y recreación(23), 5-9.

Dora, C., Hosking, J., Mudu, P., \& Fletcher, E. R. (2011). Transporte urbano y Salud. Germania: BMZ.

El Comercio. (12 de Junio de 2017). Hemos engordado más del doble desde 1980. Actualidad, págs. http://elcomercio.pe/mundo/actualidad/hemosengordado-doble-1980-senala-estudio-433882.

Fernández, Á. (2012). El potencial de las variables latentes en modelos explicativos del uso de la bicicleta. Masrid: Universidad politécnica de Madrid.

Fondo de Prevención Vial. (2006). Diagnóstico de la movilidad y la cultura ciudadana en ciudades con SITM. Bogotá: FPV.

Hilario, D. A., Núñez, A., \& Paniagua, F. R. (2015). Estudio de factibilidad de la movilidad urbana sustentable en metepec, estado de méxico 2014-2015. Mexico: Universidad Autónoma del Estado de México.

La República. (5 de Octubre de 2015). Obesidad infantil aumentó de $3 \%$ a $19 \%$ en los últimos 30 años. Salud, págs. http://larepublica.pe/impresa/sociedad/708242obesidad-infantil-aumento-de-3-19-en-los-ultimos-30anos.
Monzón, A., Rondinella , G., \& La Paix, L. (2009). Potencial del uso de la bicicleta en la ciudad de Madrid. TRANSyT, 1-16.

Morales , I. (2011). El fomento del uso de la bicicleta en entornos educativos. Huelva: WANCEULEN.

Municipalidad Metropolitana de Lima. (Julio de 2017). Propuesta de Trabajo Para Instituciones Educativas Emblemáticas. Programa "Al Cole en Bici". Lima, Lima, Perú.

Noel S., M., Santos, A., \& Taks, J. (2013). Programa UNIBICI. CDGAP, 1-7.

Pardo, C. (2006). Texto de referencia sobre transporte urbano sostenible. Eschborn.

Rivera, J. A. (2015). El uso de la bicicleta como alternativa de transporte sostenible e inclusivo para lima metropolitana. recomendaciones desde un enfoque de movilidad. Lima: Pontificia Universidad Católica del Perú.

Rodríguez P., M., Páez, D., \& Buis , J. (2017). Cómo impulsar el ciclismo urbano. Buenos Aires: BID.

Suero, D. (2010). Factibilidad del uso de la bicicleta como medio de transporte en la ciudad de Bogotá. Investigación en Ingeniería, 54-63.

Thema Equipo Editorial, S.A. (2000). Diccionario Enciclopédico llustrado. Barcelona: LEXUS. 